\title{
Dynamics of Energy Metabolism in Carbon Starvation-Induced Fruitlet Abscission in Litchi
}

\author{
Qian Wu ${ }^{1,2,+}$, Xingshuai Ma 1,2,+ , Qingxin Chen ${ }^{1,2}$, Ye Yuan ${ }^{1,2}$, Huicong Wang ${ }^{1,2}$, Xinhua He ${ }^{3}$, \\ Minglei Zhao ${ }^{1,2, *(D)}$ and Jianguo $\mathrm{Li}^{1,2, *}$
}

1 State Key Laboratory for Conservation and Utilization of Subtropical Agro-Bioresources, Ministry of Agriculture and Rural Affairs Key Laboratory of South China Horticultural Crop Biology and Germplasm Enhancement, South China Agricultural University, Guangzhou 510642, China; wuqian57q@163.com (Q.W.); maxingshuai318@126.com (X.M.); chenqingxin136@163.com (Q.C.); yuanye96210@163.com (Y.Y.); wanghc1972@263.net (H.W.)

2 Guangdong Laboratory for Lingnan Modern Agriculture, Guangzhou 510642, China

3 State Laboratory for Conservation and Utilization of Subtropical Agro-Bioresources, Guangxi University, Nanning 530004, China; honest66222@163.com

* Correspondence: zml503@scau.edu.cn (M.Z.); jianli@scau.edu.cn (J.L.); Tel.: +86-15902057353 (M.Z.); +86-13322818266 (J.L.)

+ These authors contributed equally to this work.

check for updates

Citation: $\mathrm{Wu}, \mathrm{Q} . ; \mathrm{Ma}, \mathrm{X}$.; Chen, Q.; Yuan, Y.; Wang, H.; He, X.; Zhao, M.; Li, J. Dynamics of Energy Metabolism in Carbon Starvation-Induced Fruitlet Abscission in Litchi. Horticulturae 2021, 7, 576. https://doi.org/ 10.3390/horticulturae7120576

Academic Editor: Riccardo Lo Bianco

Received: 16 November 2021

Accepted: 11 December 2021

Published: 14 December 2021

Publisher's Note: MDPI stays neutral with regard to jurisdictional claims in published maps and institutional affiliations.

Copyright: (C) 2021 by the authors. Licensee MDPI, Basel, Switzerland. This article is an open access article distributed under the terms and conditions of the Creative Commons Attribution (CC BY) license (https:/ / creativecommons.org/licenses/by/ $4.0 /)$.

\begin{abstract}
Fruit abscission is triggered by multiple changes in endogenous components of the fruit, including energy metabolism. However, it is still unknown how the core energy metabolism pathways are modified during fruit abscission. Here, we investigated the relationship between carbon starvation-induced fruitlet abscission and energy metabolism changes in litchi. The fruitlet abscission of litchi 'Feizixiao' was induced sharply by girdling plus defoliation (GPD), a carbon stress treatment. Using liquid chromatography tandem mass spectrometry (LC-MS/MS) targeted metabolomics analysis, we identified a total of 21 metabolites involved in glycolysis, TCA cycle and oxidative phosphorylation pathways. Among them, the content of most metabolites in glycolysis pathways and TCA cycles was reduced, and the activity of corresponding metabolic enzymes such as ATP-dependent phosphofructokinase (ATP-PFK), pyruvate kinase (PK), citrate synthase (CS), succinate thiokinase (SAT), and NAD-dependent malate dehydrogenase (NAD-MDH) was decreased. Consistently, we further showed that the expression of the relative genes (LcPFK2, LcPK2, LcPK4, LcCS1, LcCS2, LcSAT, $L c M D H 1$ and $L c M D H 2$ ) was also significantly down-regulated. In contrast, the level of ATP, an important metabolite in the oxidative phosphorylation pathway, was elevated in parallel with both higher activity of $\mathrm{H}^{+}$-ATPase and the increased expression level of $\mathrm{LcH}^{+}$-ATPase1. In conclusion, our findings suggest that carbon starvation can induce fruitlet abscission in litchi probably by energy depletion that mediated through both the suppression of the glycolysis pathway and TCA cycle and the enhancement of the oxidative phosphorylation pathway.
\end{abstract}

Keywords: litchi; fruitlet abscission; energy metabolism

\section{Introduction}

Litchi (Litchi chinensis Sonn.) is an important economic fruit crop widely grown in South China and Southeast Asia. According to different varieties, there will be three to five fruit drop waves during the development of fruit, which is the major cause of low yield and serious alternate bearing that greatly limits the healthy development of the litchi industry [1]. Thus, it is of great significance to explore the regulation mechanism of litchi fruit abscission, which would help to improve current cultural and management practices.

Glycolysis, tricarboxylic acid cycle and oxidative phosphorylation are three major energy metabolism pathways during plant development. The glycolysis pathway (EMP), the oxidization of glucose to pyruvate and produce ATP, is a common pathway of glucose degradation in all biological organisms [2]. The tricarboxylic acid cycle, also known as 
the Krebs cycle or TCA, occurs in mitochondria [3]. TCA is the hub and final metabolic pathway of carbohydrate, lipid, and amino acid. The oxidative phosphorylation pathway (OXPHOS) refers to the process by which mitochondria uses the energy generated from the oxidation process of sugar, lipid and amino acid to produce adenosine triphosphate (ATP) by promoting the combination of adenosine diphosphate (ADP) and inorganic phosphate [4]. Through these energy metabolism pathways, nutrients are transformed into various metabolites, and ATP is produced in plants, thus providing sufficient energy for plant growth and development [5-7].

In plants, the energy metabolism pathways, such as EMP, TCA and OXPHOS, are changed significantly under stress conditions, and the change patterns are different in individual plants under different environments. Liu et al. [8] analyzed sugar beet roots under salt stress by transcriptome and metabolome techniques, and found that both the content of most metabolites and the expression level of genes encoding enzymes involved in the EMP and TCA cycle were increased, indicating that the EMP and TCA cycle are enhanced in response to salt stress. The activity of ATP synthase, fructose diphosphate aldosterone, and malate dehydrogenase was also significantly increased in bermudagrass under drought stress [9]. In contrast, the content of glucose-6-phosphate, fructose-6-phosphate, succinic acid, fumaric acid and malic acid was significantly reduced in barley buds and roots under phosphorus deficiency, suggesting that barley could adapt to a low level of phosphorus by regulating the EMP and TCA [10]. By metabolomics analysis on wheat leaves under drought stress, Guo et al. [11] also found that the content of malic acid, citric acid, aconitic acid, and other metabolites involved in the TCA cycle was significantly reduced, suggesting that the TCA cycle might be suppressed in wheat under drought stress. In addition, the energy metabolism of peach and banana under low temperature after harvest was deprived as the activity of $\mathrm{H}^{+}$-ATPase, succinic dehydrogenase, and ATP content was decreased [12,13]. Collectively, these findings suggest that the energy metabolism pathways show different change patterns in response to different stress conditions. However, it remains poorly understood how the energy metabolism pathways are altered during fruit abscission.

In the study of plant organ abscission, it was found that carbon stress (insufficient carbon supply) could lead to massive organ abscission. For example, decreased carbon assimilation by shading treatment would induce the abscission of apple fruit and grape flower [14,15]. In litchi and longan, carbon starvation induced by girdling resulted in a large amount of fruit abscission [16,17]. What's more, there has been increased evidence that energy metabolism was changed during the process of organ abscission caused by carbon stress. Domingos et al. [18] found that the expression of ATPase related genes was significantly up-regulated in shading-treated grapevine flower organs. Meanwhile, genes related to EMP, such as those encoding pyruvate dehydrogenase and citrate synthase, were down-regulated in the process of litchi fruit abscission induced by carbon stress [19]. These results imply that energy metabolism is altered during the process of fruit abscission caused by carbon stress. However, the precise changes of energy metabolism pathways involved in carbon stress-induced fruit abscission are yet unknown.

Metabolomics can be used to qualitatively analyze all the small molecule metabolites in cells at a given time and environment condition, so as to quantitatively describe the whole body of endogenous metabolites and their response to changes in the internal and external factors [20]. At present, metabolomics has been widely used in the study of plant responses under stress conditions. In this study, LC-MS/MS targeted metabolomics was used to analyze the energy metabolites in the process of litchi fruitlet abscission induced by carbon stress. Additionally, we further determined the activity of the major metabolic enzymes involved in these metabolites and the expression of genes encoding these enzymes during fruitlet abscission. This study provides new insight into the multi-dimensional changes of energy metabolism pathways in carbon stress induced litchi fruitlet abscission, including gene expression, enzyme activity, and metabolite content. 


\section{Materials and Methods}

\subsection{Plant Material and Treatments}

Three randomly selected 16-year-old litchi trees (Litchi chinensis Sonn.cv. Feizixiao) grown in a Xili orchard (Shenzhen, China) were chosen. One plant was a biological repeat, and twenty fruit-bearing shoots with similar diameter (diameter of about $5 \sim 8 \mathrm{~mm}$ ) located in different directions from each tree were tagged. Ten of them were treated with girdling (a ring of bark about $0.5 \mathrm{~cm}$ in width and cambium was removed from the branch base) followed by defoliation (removing all leaves above the girdle) at 35 days after anthesis (GPD treatment), while the other untreated shoots were used as control (CK). Among these three sets, three of each were used to monitor the fruit abscission dynamic and the others were sampled for further experiments. The collected samples were quickly frozen in liquid nitrogen and stored at $-80{ }^{\circ} \mathrm{C}$ for future analysis.

\subsection{Measurement of Energy Metabolites}

Litchi fruitlet retrieved from $-80^{\circ} \mathrm{C}$ storage were ground into fine powder in liquid nitrogen. Powder $(60 \mathrm{mg})$ was vortexed in a $1 \mathrm{~mL}$ solution of methanol/acetonitrile $/ \mathrm{H}_{2} \mathrm{O}$ $(2: 2: 1, v / v / v)$. After sonication for $30 \mathrm{~min}$ on ice, the mix was stored at $-20{ }^{\circ} \mathrm{C}$ for 1 $\mathrm{h}$ to precipitate proteins. The mix was centrifuged for $20 \mathrm{~min}\left(14,000 \mathrm{rpm}, 4^{\circ} \mathrm{C}\right)$ and dried by a vacuum drying system. A targeted metabolic analysis was performed using a LC-MS/MS system. The dried metabolites were dissolved in $100 \mu \mathrm{L}$ of acetonitrile/ $\mathrm{H}_{2} \mathrm{O}(1: 1, v / v)$ and centrifuged for $15 \mathrm{~min}\left(14,000 \mathrm{rpm}, 4^{\circ} \mathrm{C}\right)$. Finally, the supernatant was sampled for analysis. Electrospray ionization was conducted with an Agilent 1290 Infinity chromatography system and AB Sciex QTRAP 5500 mass spectrometer. Ammonium acetate solution $(15 \mathrm{mM})$ and acetonitrile were used as mobile phases $A$ and $B$, respectively. The sample was placed in a $4{ }^{\circ} \mathrm{C}$ automatic sampler, the column temperature was $45^{\circ} \mathrm{C}$, the flow rate was $300 \mu \mathrm{L} / \mathrm{min}$, and the injection volume was $4 \mu \mathrm{L}$. A relative liquid gradient was used as follows: the gradient for B was 0-18 $\mathrm{min}$ at $90 \%$ to $40 \% ; 18-18.1 \mathrm{~min}$ at $40 \%$ to $90 \%$; and $18.1-23 \mathrm{~min}$ at $90 \%$. In the sample queue, a QC sample was set for each interval of a certain number of experimental samples, which was used to detect and evaluate the stability and repeatability of the system; the standard mixture of energy metabolism substances was set up in the sample queue to correct the retention time of chromatography. The AB Sciex QTRAP 5500 mass spectrometer was operated in the negative mode under the following conditions: source temperature $450{ }^{\circ} \mathrm{C}$, ion Source Gas (Gas1): 45, Ion Source Gas2 (Gas2): 45, Curtain gas (CUR): 30, ion Sapary Voltage Floating (ISVF): -4500 V; MRM mode was used to detect the ion pairs to be measured.

\subsection{Enzyme Activity Analysis}

Extraction of enzyme solution: fruit sample $(0.1 \mathrm{~g})$ was ground thoroughly, then added $1.8 \mathrm{~mL}$ of precooled extraction buffer (100 mM Hepes- $\mathrm{NaOH}$ (pH7.5), $5 \mathrm{mM} \mathrm{MgCl}_{2}, 1 \mathrm{mM}$ EDTA, $2.5 \mathrm{mM}$ DTT, 0.5\% TritonX-100, 1\% BSA, 5\% PVPP and 10\% glycerol). Next, the homogenate was centrifuged at $13,000 \mathrm{rpm}$ for $10 \mathrm{~min}$ at $4{ }^{\circ} \mathrm{C}$, and the supernatant was collected for enzyme activity examination.

The activity of NAD-dependent malate dehydrogenase (NAD-MDH), citrate synthase (CS), fumarase (FUM) was determined according to the method described by Jenner et al. [21]. The activity of ATP-dependent phosphofructokinase (ATP-PFK), pyruvate kinase (PK) was examined according to the method described by Burrell et al. [22]. The activity of succinate thiokinase (SAT) was tested according to the method of Danson et al. [23]. The activity of succinate dehydrogenase (SDH) activity was determined by the method of Schirawski and Unden [24]. The activity of $\mathrm{H}^{+}$-ATPase activity was determined according to the method of Zhang et al. [25].

\subsection{RNA Extraction}

Total RNA was extracted from litchi fruitlet using an ultrafast plant RNA Extraction Kit (Huayueyang, Beijing, China) according to the instructions. RNA concentration and purity 
was determined by a NANODROP 2000 ultramicro spectrophotometer (USA). The cDNA synthesis was generated using $2 \mu \mathrm{g}$ total RNA according to the manual of the TransScript One-Step gDNA Removal and cDNA Synthesis SuperMix Kit (TransGen, Beijing, China).

\subsection{Real-Time PCR Analysis}

Energy metabolism relative genes were identified from the litchi genome database (http:/ /121.37.229.61:82/, accessed on 1 January 2021). Next, primers were designed with primer 5.0 (see Table 1 for the primer sequence). Bio-Rad ABI 7500 Real-Time PCR System (Bio-Rad, Hercules, CA, USA) was used for real-time quantitative PCR. The program was set as follows: pre denaturation at $95^{\circ} \mathrm{C}$ for $5 \mathrm{~min}$, denaturation at $95^{\circ} \mathrm{C}$ for $10 \mathrm{~s}$, annealing at $55^{\circ} \mathrm{C}$ for $30 \mathrm{~s}$, extending for at $72{ }^{\circ} \mathrm{C} 30 \mathrm{~s}$, and for 40 cycles, followed by melting curve analysis. Each sample was quantified at least in triplicate and expression normalized against that of EF-1a as an internal control [26]. The relative expression level of target genes was calculated using the $2^{-\Delta \Delta C T}$ method.

Table 1. Primer sequences used in this study.

\begin{tabular}{|c|c|c|}
\hline Name & Forward Primer $\left(5^{\prime}-3^{\prime}\right)$ & Reverse Primer $\left(3^{\prime}-5^{\prime}\right)$ \\
\hline LCPFK & AGACTGCTTTCTTCTACCAACC & TCAGACTTTCTCCATCCAACA \\
\hline LcPK1 & GGAGTTTTAGTGGAGCCTTTG & TTGTAGACTCGGCAGGATGT \\
\hline LcPK2 & TCGCAATCAAAGCGTCCG & TTCCCACTCACCTGCGTCA \\
\hline LcCS1 & GCGGCTGCGTTAAATGGT & GCTCCTTGGTTGCGTTCTC \\
\hline LcCS2 & ATTCGGGTGGTGGCTATTA & ATGGTTCCAGCAGTGTCGC \\
\hline$L c S D H$ & СTCTTCССТCTTGGTCCTCC & GGTTAGCAACTTCCTGTCCG \\
\hline LCSAT & AGGAGTCGCTGCTTCTTCG & CCACCCTTGAGACCACTTTTA \\
\hline LcFUM & CGGTGAGATTGCTGGGTGA & CCTTGTGGGCTTTCTTTGC \\
\hline LcMDH1 & GTTGTCACTTCTCAAATCCGTC & TCTGATCCAGAAAATTGTGCTGACT \\
\hline LcMDH2 & GATGGAGGGACAGAAGTCGT & AAGGTAATTCGGTGATGGTTG \\
\hline $\mathrm{LcH}^{+}$-ATPase 1 & GTTCATCATCCGCTACGCTT & СТСССТTTCСATAGTCTTTCTTG \\
\hline $\mathrm{LcH}^{+}$-ATPase 2 & AGTTCGATTTCTCGCCTTTC & TGGATCTTACACCGAATTTGTC \\
\hline
\end{tabular}

\subsection{Data Analysis}

The chromatographic peak area and retention time were extracted by multiquant software. The retention time was corrected by the standard substance of energy metabolism substance, and the metabolites were identified. Significant differences were determined by one-way analysis of variance (ANOVA) using SPSS W version 13.0 (SPSS, Inc., Chicago, IL, USA), while statistical differences were assessed with a significance level of $5 \%$. Graphs were drawn with SigmaPlot 10.0 and Excel 2003.

\section{Result}

\subsection{Carbon Starvation Induced Fruitlet Abscission in Litchi}

We have previously shown that GPD treatment can induce carbon starvation in litchi fruitlet in a short time period [19]. Consistent with this, the cumulative fruitlet abscission rate was significantly increased after GPD treatment. On the second day, the cumulative abscission rate of GPD-treated fruitlets was $53.76 \%$, while it was only $18.33 \%$ in the control fruitlet. On the third day, $100 \%$ of GPD-treated fruitlets dropped; in contrast, only $23.74 \%$ of control fruitlets dropped (Figure 1). These results indicate that GPD-induced carbon starvation can activate litchi fruitlet abscission in a couple of days.

\subsection{Effect of Carbon Starvation on Energy Metabolites in Litchi Fruitlet}

In order to examine how the energy metabolites were changed in GPD-treated fruitlets, we performed LC-MS/MS targeted metabolomics. As shown in Figure 2, the RSD (relative standard deviation) of the detected energy metabolites, except NADP, was less than $30 \%$, indicating that the detection instrument used in this experiment has good stability and high data quality, which can be used for further analysis. In total, 21 energy metabolites related to EMP, TCA cycle and OXPHOS were identified. 


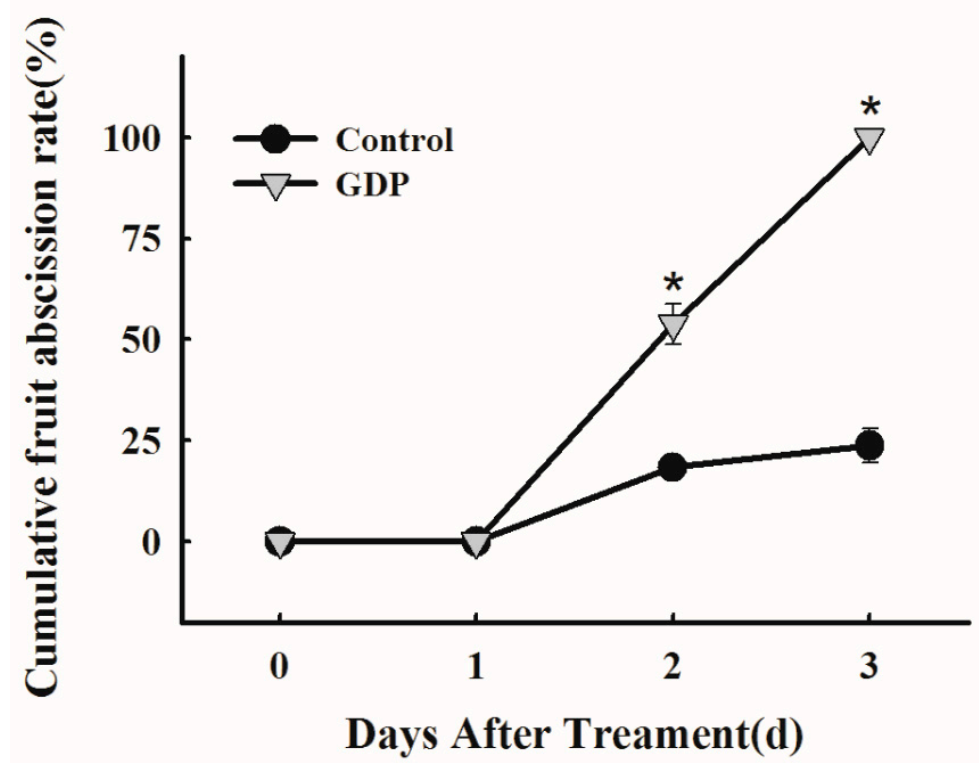

Figure 1. The effect of starvation stress on fruitlet abscission of litchi. Each value represented the means of three biological replicates from three different trees, with the SE indicated by vertical bars. And $p<0.05(*)$ according to student $t$-test was considered as significant differences.

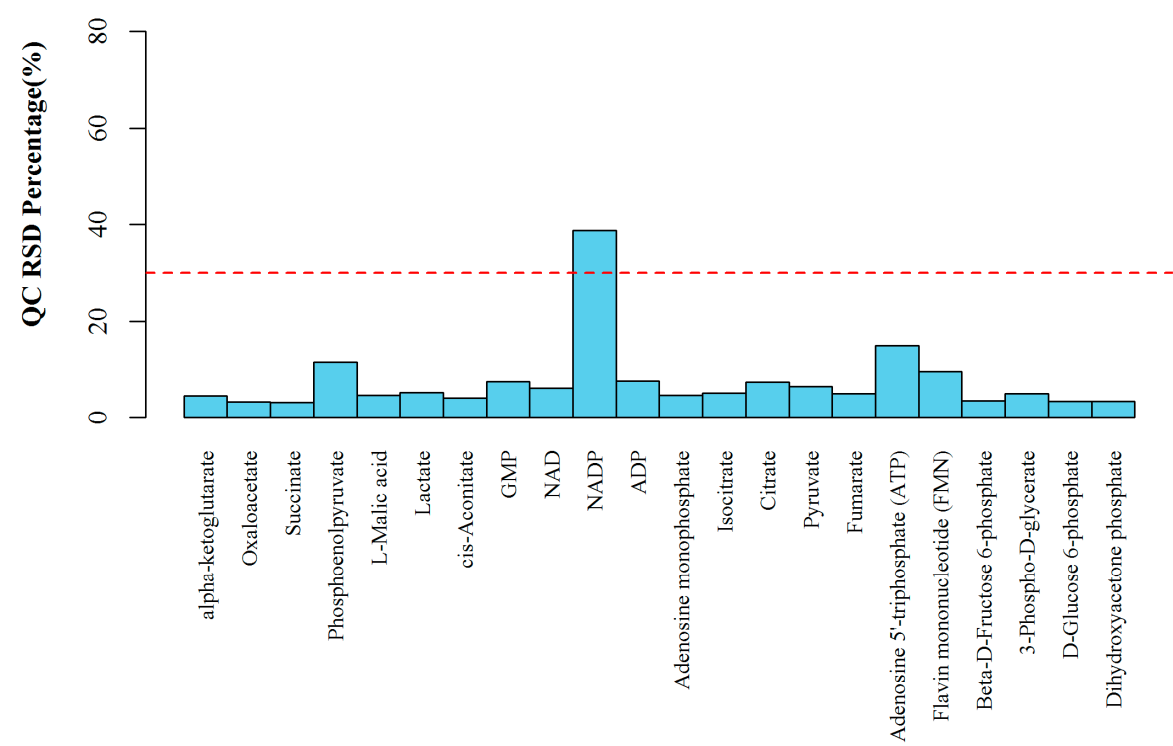

Figure 2. The RSD distribution of QC samples. All the samples were mixed into quality control (QC) samples. The stability and repeatability of the data were evaluated by QC samples. The smaller the RSD (RSD < 30\%) of the substance to be tested in QC samples indicates that the metabolite is relatively stable and less affected by the matrix effect, and the quantitative value of each metabolite can reflect the difference.

For EMP, a total of seven metabolites were detected, including glucose-6-phosphate (G6P), fructose-6-phosphate (F6P), dihydroxyacetone phosphate (DHAP), 3-phosphoglycerate (3GP), phosphoenolpyruvate (PEP), pyruvate, and lactic acid. As shown in Figure 3 , the content of both 3GP and PEP was slightly increased at day 1, but decreased from day 2, and reached a significant low level at day 3 after GPD treatment. Similarly, pyruvate content in GPD-treated fruitlet was significantly induced at day 1, but significantly decreased at day 2 and day 3 compared to that of control. In addition, the DHAP level decreased greatly after GPD treatment at day 2 and day 3. Taken together, these results indicate that the content of most metabolites (3PG, PEP, DHAP, pyruvate) related to EMP were significantly decreased 
in GPD-treated litchi fruitlets. However, the decreases observed do not correspond to the timing of GPD induced abscission.

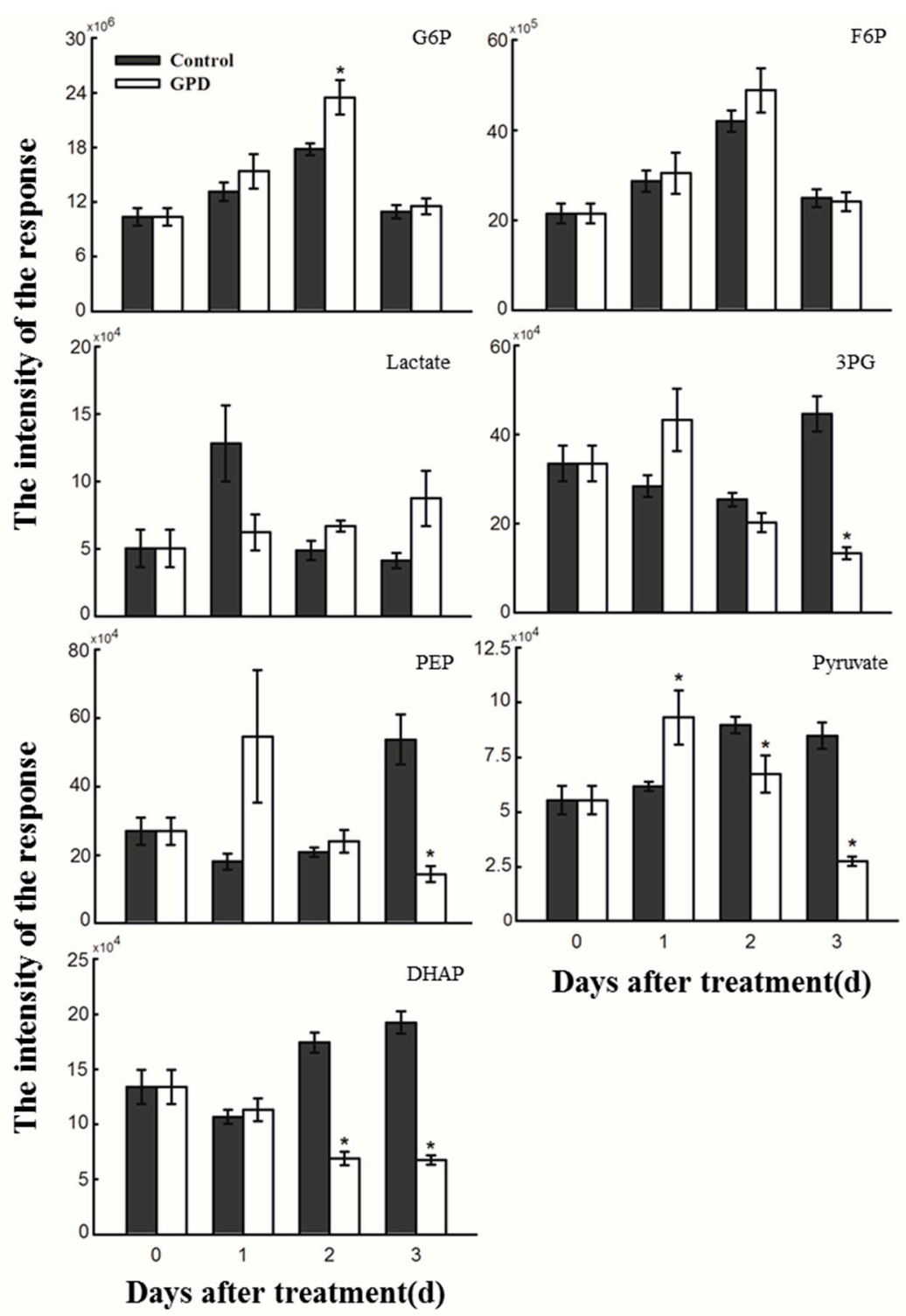

Figure 3. The effect of starvation stress on the content of metabolites related to glycolysis. The response intensity value represents the response intensity of relevant metabolites on the instrument. Values were expressed as the means $\pm \mathrm{SD}$ of triplicate assays, and $p<0.05(*)$ according to the Student's $t$-test was considered as significant differences.

For the TCA cycle, six metabolites including citric acid, cis-aconitate, isocitrate, $\alpha$ ketoglutarate, malic acid and oxaloacetate were identified. As shown in Figure 4, the content of five metabolites including isocitrate, citrate, cis-aconitate, $\alpha$-ketoglutarate and malic acid was significantly decreased, of which, its content, from day 1 in GPD-treated fruitlet, except for isocitrate, whose content was decreased at day 3 only. Collectively, these findings show that the level of most metabolites related to the TCA cycle were remarkably decreased in litchi fruitlet from day 1 after GPD treatment, suggesting that the TCA cycle was suppressed prior to the occurrence of GPD induced fruit abscission in litchi. 


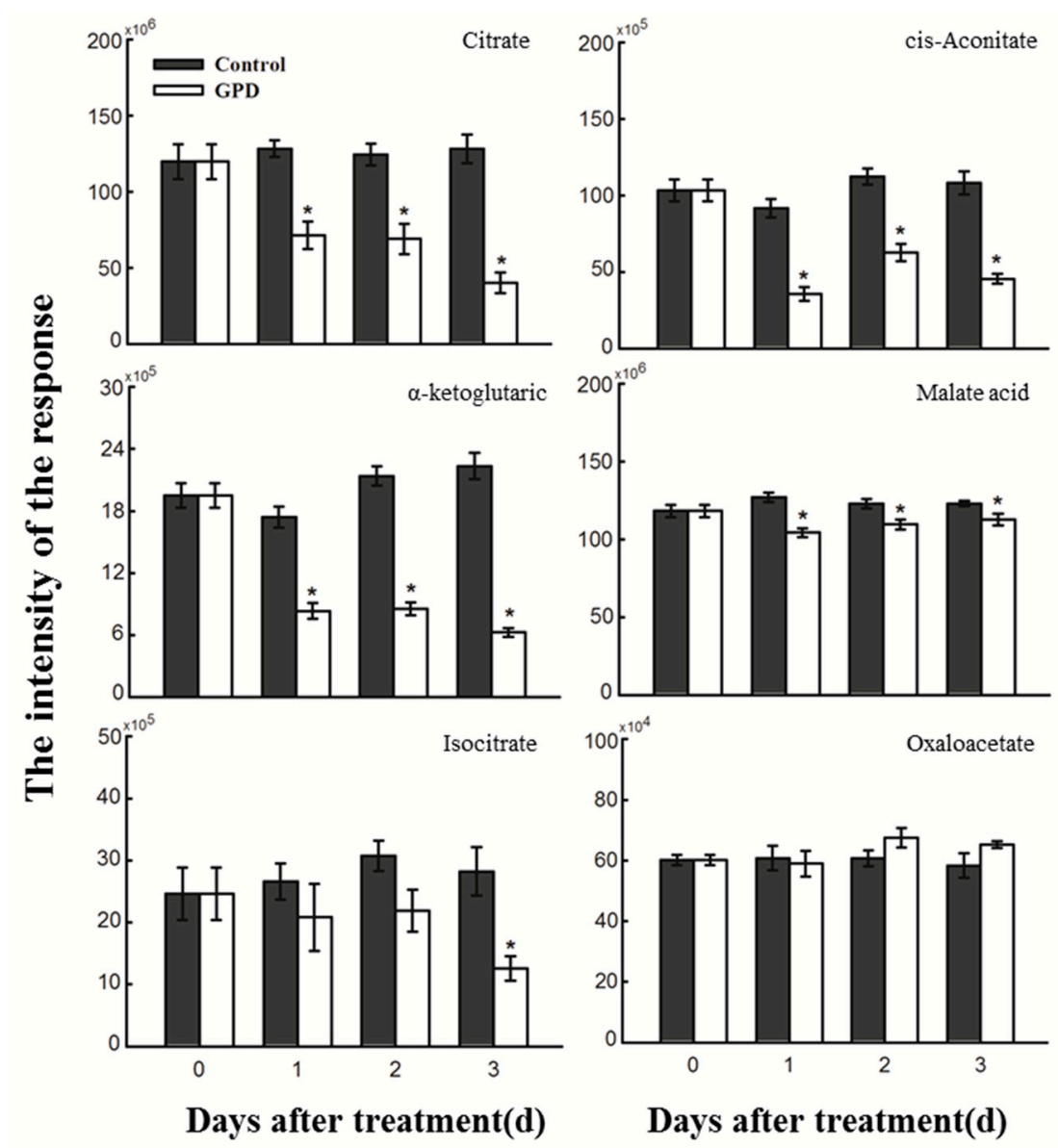

Figure 4. The effect of starvation stress on the content of metabolites related to TCA cycle. The response intensity value represents the response intensity of relevant metabolites on the instrument. Values were expressed as the means \pm SD of triplicate assays, and $p<0.05(*)$ according to student $t$-test was considered as significant differences.

For OXPHOS, seven metabolites, including succinic acid, fumaric acid, adenosine triphosphate (ATP), adenosine diphosphate (ADP), adenosine acid (AMP), flavin mononucleotide (FMN) and nicotinamide adenine dinucleotide (NAD), were identified. As shown in Figure 5, the content of succinic acid was significantly decreased, while the fumaric acid content was increased from day 2 in GPD-treated fruitlet compared to that of the control. As an important metabolite of OXPHOS, ATP content was increased remarkably in GPD-treated fruitlet at day 1 and day 2. And the level of AMP and ADP was also significantly increased from day 1 or at day 2 in GPD-treated fruitlets, respectively. In addition, the level of both FMN and NAD was significantly higher from day 2 after GPD treatment. Together, these results indicate that OXPHOS was enhanced in GPD-treated litchi fruitlets. 


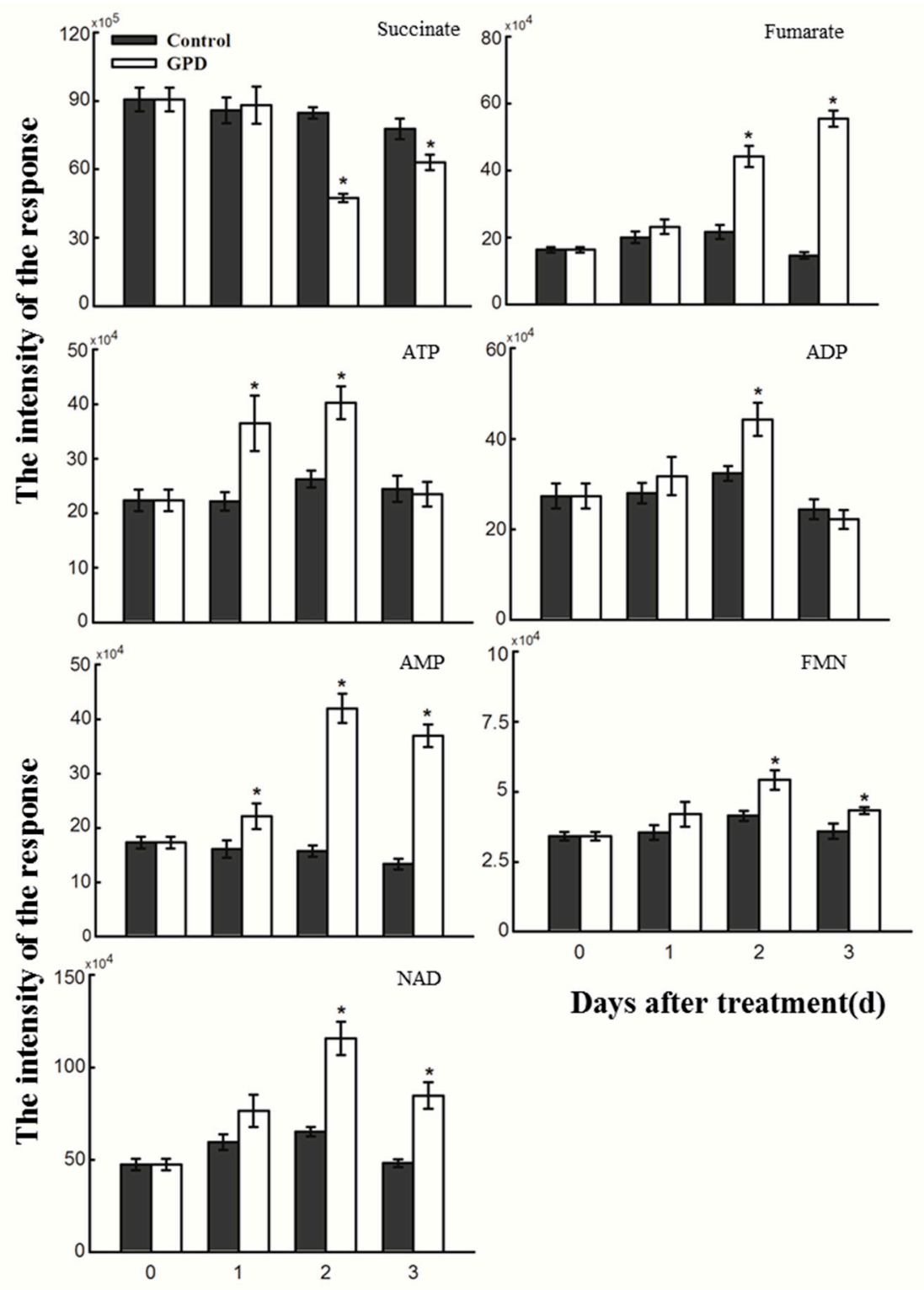

Days after treatment(d)

Figure 5. The effect of starvation stress on the content of metabolites related to oxidative phosphorylation pathways. The response intensity value represents the response intensity of relevant metabolites on the instrument. Values were expressed as the means \pm SD of triplicate assays, and $p<0.05(*)$ according to student $t$-test was considered as significant differences.

\subsection{Effect of Carbon Starvation on the Activity of Enzymes Related to Energy Metabolism}

PK and ATP-PFK are two key regulatory enzymes in the EMP metabolic process. ATP-PFK catalyzes fructose-6-phosphate into fructose 1,6-diphosphate, and PK catalyzes the conversion of phosphoenolpyruvate to pyruvate. We found that the activity of both PK and ATP-PFK was significantly reduced from day 1 and 2, and their activity was reduced by $50.92 \%$ and $44.03 \%$ after GPD treatment at day 2, respectively (Figure $6 \mathrm{~A}, \mathrm{~B}$ ). 
EMP

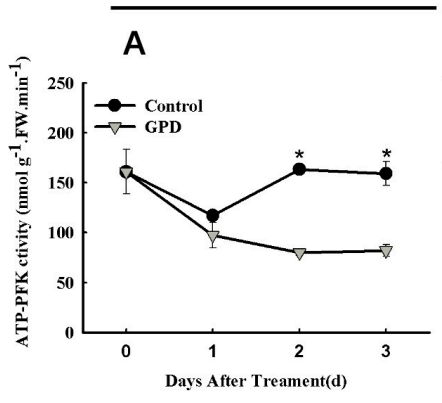

B

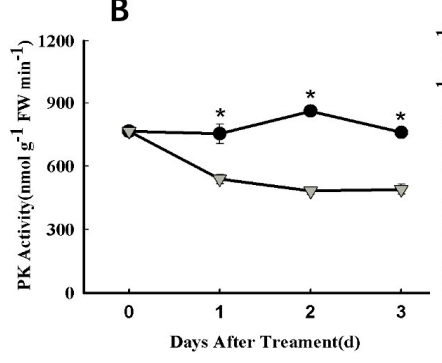

TCA
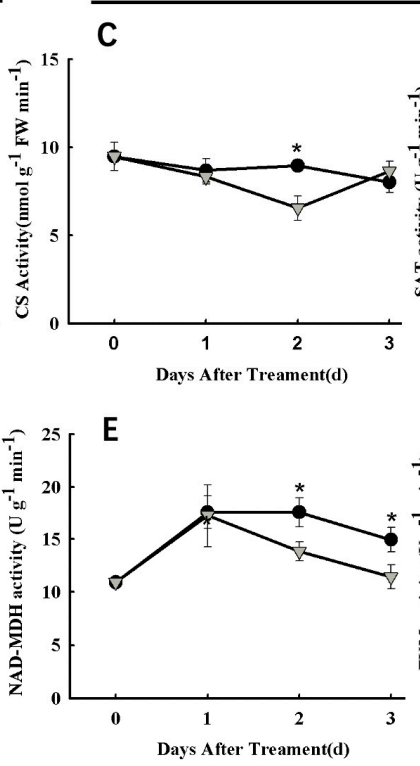

D

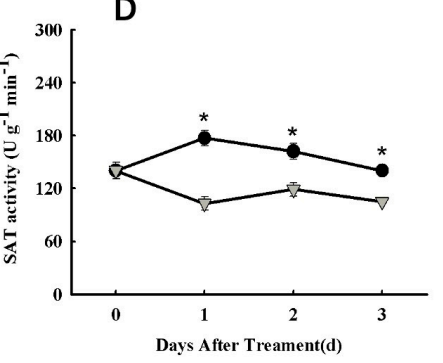

$\mathbf{F}$

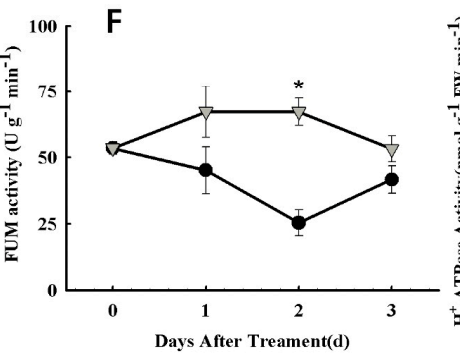

OXPHOS
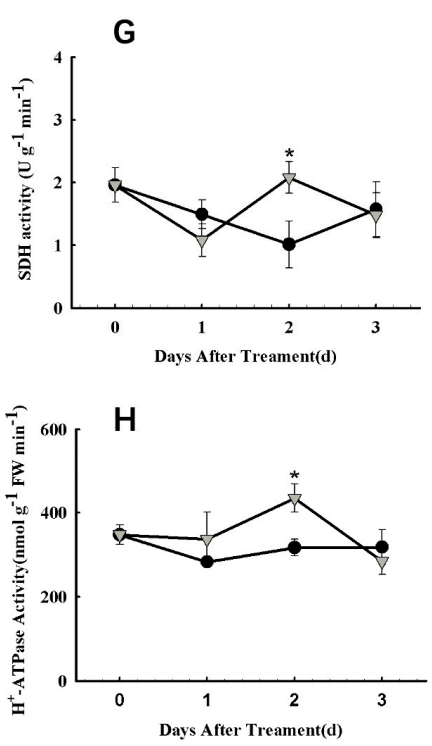

Figure 6. The effect of starvation stress on the activity of enzymes related to energy metabolism. ATP-dependent phosphofructokinase (A), Pyruvate kinase (B), Citrate synthase (C), Succinate thiokinase (D), NAD-dependent malate Dehydrogenase (E), Fumarase $(\mathbf{F})$, Succinate dehydrogenase $(\mathbf{G}), \mathrm{H}^{+}$-ATPase $(\mathbf{H})$. Values were expressed as the means $\pm \mathrm{SD}$ of triplicate assays, and $p<0.05(*)$ according to student $t$-test was considered as significant differences.

In the TCA cycle, CS is the first enzyme that catalyzes the synthesis of citric acid from oxaloacetate and acetyl-CoA. As shown in Figure 6C, CS activity was first decreased and then increased after GPD treatment, with its obviously lower activity at day 2. SAT catalyzes the formation of succinyl-CoA to succinic acid and coenzyme A, while NADMDH catalyzes the conversion of malic acid to oxaloacetate. Similarly, the activity of both SAT and NAD-MDH was also significantly decreased in fruitlet after GPD treatment, with a significant reduction of $41.89 \%$ SAT activity in GPD-treated fruitlet at day 1 (Figure 6D,E). In contrast, the activity of FUM, which catalyzes fumaric acid to oxaloacetate, was increased to $164.96 \%$ in GPD-treated fruitlet at day 2 compared to that of control (Figure 6F).

$\mathrm{SDH}$, which catalyzes the conversion of succinic acid to fumaric acid, is a key enzyme in OXPHOS. SDH activity was found to be significantly increased by $105.94 \%$ in GPDtreated fruitlets compared to that in control fruitlets (Figure 6G). Another key enzyme, $\mathrm{H}^{+}$-ATPase, can consume ATP to transfer hydrogen ions directionally; its activity saw a significant increase of $36.59 \%$ after GPD treatment at day 2 (Figure $6 \mathrm{H}$ ).

\subsection{The Expression of Genes Related to Energy Metabolism in GPD-Treated Fruitlet in Litchi}

Given that the activity of enzymes related to energy metabolism was changed in GPD-treated fruitlet in litchi, we thus hypothesized that the expression of genes encoding these enzymes might also be changed. To test this, we carried out qRT-PCR analysis on the expression of 12 genes that encode enzymes involved in EMP, TCA, and OXPHOS. Among these 12 genes, one ATP-PFK (named LcPFK) and two PK genes (named LCPK1-2) that were related to EMP were significantly down-regulated in GPD-treated fruitlets from day 1 (Figure 7).

For the TCA cycle, two CS (named LcCS1-2), one SAT (named LcSAT), one FUM (LcFUM), and two NAD-MDH genes (named LcMDH1-2) were investigated. We found that the expression of $L c C S 1, L c C S 2, L c M D H 1$ and $L c M D H 2$ was greatly repressed from day 1 after GPD treatment, of which LcCS2 was the most inhibited gene. The level of LcSAT transcripts was also significantly lower in GPD-treated fruitlet than that in control fruitlet at day 1 and day 3 . In contrast, the expression of LcFUM in fruitlet was increased after GPD treatment with a peak at day 1. 

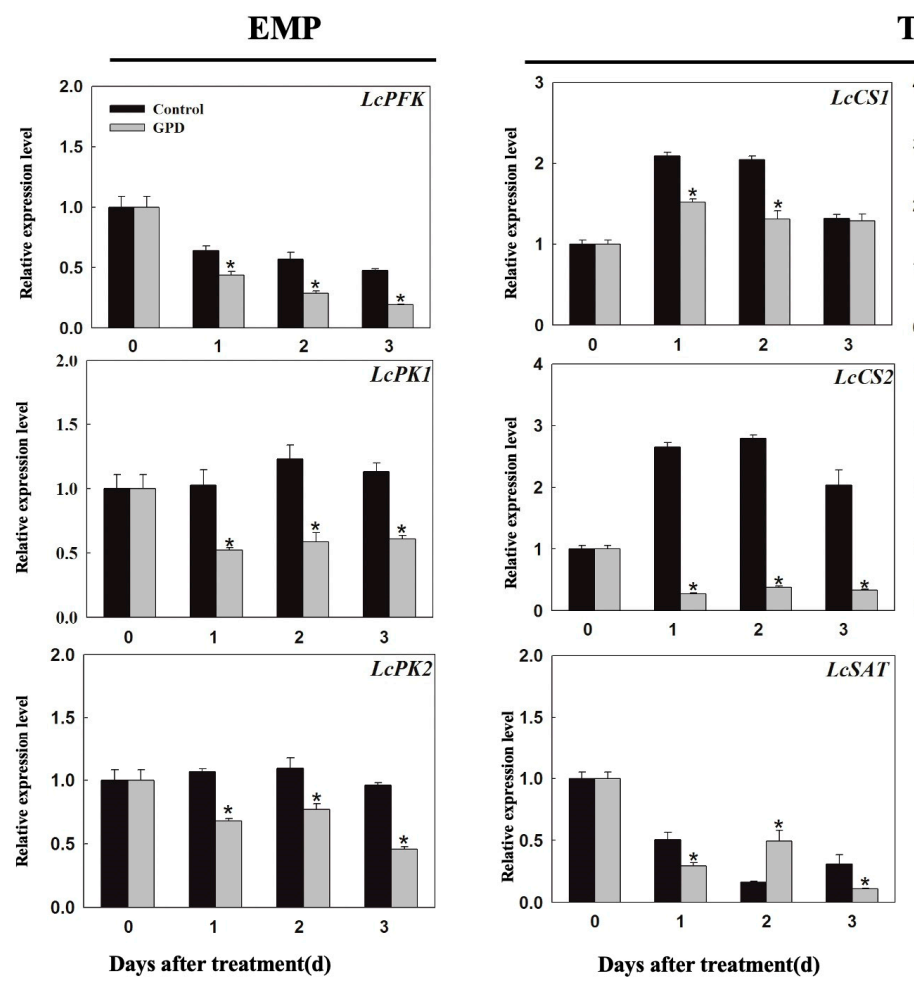

TCA
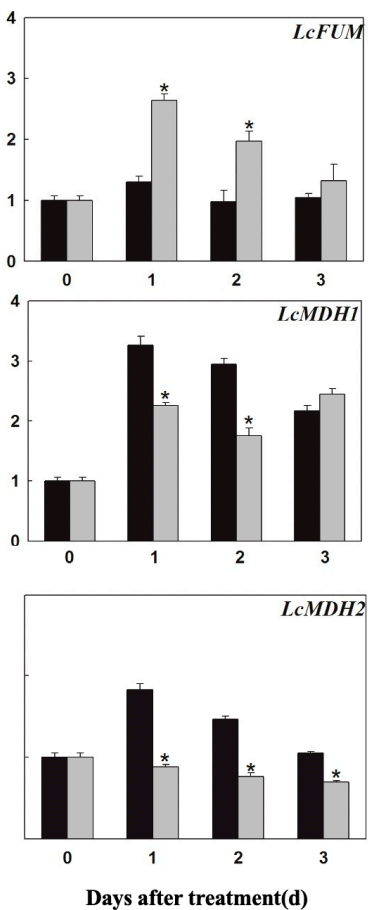
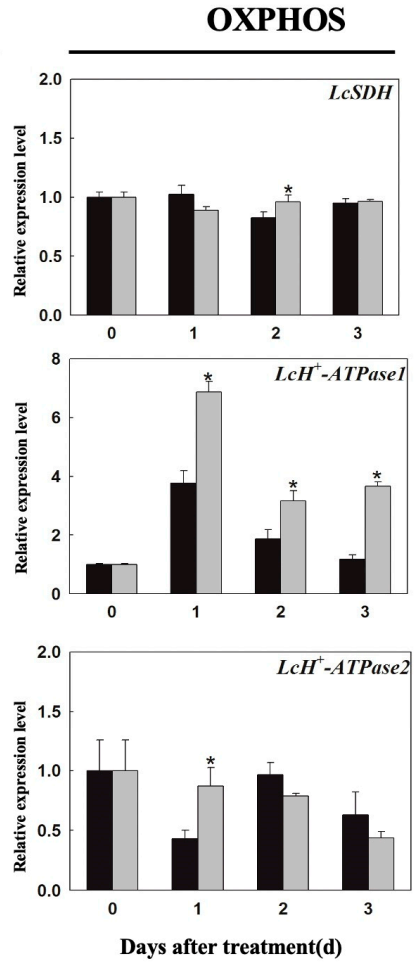

Figure 7. The effect of starvation stress on the expression of genes related to energy metabolism. Transcript abundance was determined using qRT-PCR. The value of transcript levels in control was set to 1 . Values were expressed as the means $\pm \mathrm{SD}$ of triplicate assays, and $p<0.05(*)$ according to student $t$-test was considered as significant differences.

A total of $1 \mathrm{SDH}$ (named $L c S D H$ ) and $2 \mathrm{H}^{+}$-ATPase genes (named $\mathrm{LcH}^{+}$-ATPase1-2) involved in OXPHOS were identified from the litchi genome. As shown in Figure 7, the expression level of $L c S D H$ was significantly higher in GPD-treated fruitlets than that in the control fruitlet at day 2. Additionally, $\mathrm{LcH}^{+}$-ATPase 1 was significantly increased from day 1 after GPD treatment, while the expression of $\mathrm{LcH}^{+}$-ATPase2 was significantly up-regulated at day 1 after GPD treatment.

\section{Discussion}

Throughout the fruit development, the excessive fruit abscission is a major problem that causes serious economic loss for the growers. Previous studies have revealed that endogenous hormone changes and carbohydrates in short supply are the main factors for fruit abscission [17,27-29]. Carbohydrates, as the source of energy, play an indispensable role in plant growth and development [30]. In this study, the fruitlet abscission was initiated from day 1, and $100 \%$ of litchi fruitlet dropped at day 3 after carbon starvation treatment (Figure 1).

EMP is an important pathway of energy metabolism, with its main function in converting sucrose into pyruvate and producing energy substance ATP in plant cells. In this study, we detected seven energy metabolites related to EMP in litchi fruitlets after GPD treatment. We found that the EMP metabolites were significantly down-regulated after GPD treatment. Particularly at day 3 when the abscission had been initiated, four metabolites (3PG, PEP, pyruvate and DHAP) were apparently decreased in GPD-treated fruitlets compared to that in the control fruitlets (Figure 3). In general, the metabolic enzyme activity is responsible for the corresponding metabolite level [31]. PK, as key regulatory enzyme of EMP, determines the concentration of PEP by catalyzing the terminal reaction of EMP. ATP-PFK is the most important rate-limiting enzyme in the oxidation process of sugar, and the reaction is irreversible [32]; the change of ATP-PFK activity will cause significant subsequent changes in the content of metabolites such as G6P [22]. Our findings showed that the activity of both ATP-PFK and PK was significantly decreased in 
GPD-treated fruitlets. Interestingly, the expression of genes encoding ATP-PFK and PK, such as $L c P F K, L c P K 1$ and $L c P K 2$, was significantly down-regulated before the abscission had been initiated. Therefore, we propose that under carbon starvation, the expression of LcPFK, LcPK1, and LcPK2 is down-regulated, which causes a low activity of both ATP-PFK and PK, leading to the reduction of related metabolites in EMP after the abscission had been initiated.

The TCA cycle is the most effective way to gain energy through driving the oxidation of sugar nutrients, which also acts as the main energy source during plant life [33]. In our study, a total of seven energy metabolites related to the TCA cycle were detected in litchi fruitlets. Similar to the changes of EMP metabolites in litchi fruitlets under carbon starvation, the TCA metabolites were decreased after GPD treatment. At day 2 when the abscission had been initiated, the content of four metabolites, including citric acid, cisaconitate, isocitrate, and malic acid, was significantly decreased. Decrease in the content of these metabolites might be due to a low activity of CS, SAT, and NAD-MDH (Figure 6 C-E). We found that their activity, including CS, SAT, and NAD-MDH, were inhibited when the abscission had been initiated by carbon starvation treatment, which was consistent with the results of metabolite content changes. Furthermore, we found that the expression of relative genes including LcCS1, LcCS2, LcSAT, LcMDH1 and LcMDH2 was significantly downregulated before the abscission had been initiated (Figure 7), supporting the decreased activity of enzymes encoded by these genes. Together, these findings indicate that the TCA cycle is suppressed when the abscission had been initiated in GPD-treated fruitlets.

OXPHOS, which occurs in mitochondria or bacteria of eukaryotic cells, is the main pathway of ATP production [34]. OXPHOS is a process by which substances release energy during oxidation to produce ATP via coupling ADP and inorganic phosphorus. In our study, we found that the level of fumaric acid, FMN, NAD, AMP and ATP was greatly elevated before or when the abscission had been initiated. $\mathrm{H}^{+}$-ATPase is a key enzyme in mitochondrial respiration and metabolism, and its activity directly affects the production of energy substance ATP [25]. Here, we showed that the level of ATP was consistent with the $\mathrm{H}^{+}$-ATPase activity, which was further correlated with the expression level of $\mathrm{LcH}^{+}$-ATPase1 in GPD-treated fruitlets before the abscission had been initiated. In addition, as one enzyme that locates at the mitochondrial inner membrane, SDH is not only involved in TCA cycle, but also plays important roles in OXPHOS. We found that SDH activity was significantly increased at day 2, probably due to the up-regulated expression $L c S D H$ in GPD-treated fruitlets. Based on these results, we speculated that OXPHOS was enhanced when the abscission had been initiated in litchi fruitlet under carbon starvation.

In conclusion, we proposed a model that illuminates a role of energy metabolics in litchi fruitlet abscission under carbon starvation (Figure 8). At day 1 before the abscission had been initiated after GPD treatment, the level of metabolites related to EMP, TCA and OXPHOS was less affected, except for ATP and AMP. At day 2 when the abscission had been initiated, metabolites involved in both EMP and TCA cycle were significantly decreased. Whereas both the ATP content and the activity of $\mathrm{H}^{+}$-ATPase and SDH in the OXPHOS pathway were dramatically enhanced. At day 3, metabolites related to the EMP and TCA cycle continued to decrease. Interestingly, it seems to be a very strong transcriptional response, since most genes related to EMP, TCA and OXPHOS were significantly changed before the abscission had been initiated, compared with metabolite and enzyme activity assays, which suggests that there are other genes that may be affected that are more important than those related to carbon metabolism, since the changes in the enzyme activity and metabolites come later. While these genes are part of the response, there may be others with altered expression that are more important to initiate abscission. Together, we speculate that carbon starvation will trigger OXPHOS, which allows fruitlets to consume the stored substrate and produce a large amount of the energy substance ATP in a short period of time as a stress response. However, the EMP and TCA cycle are suppressed in parallel, which leads to the failure of new substrate supply for energy synthesis, thereby aggravating the exhaust of energy in GPD-treated fruitlets and accelerating fruitlet abscission. 


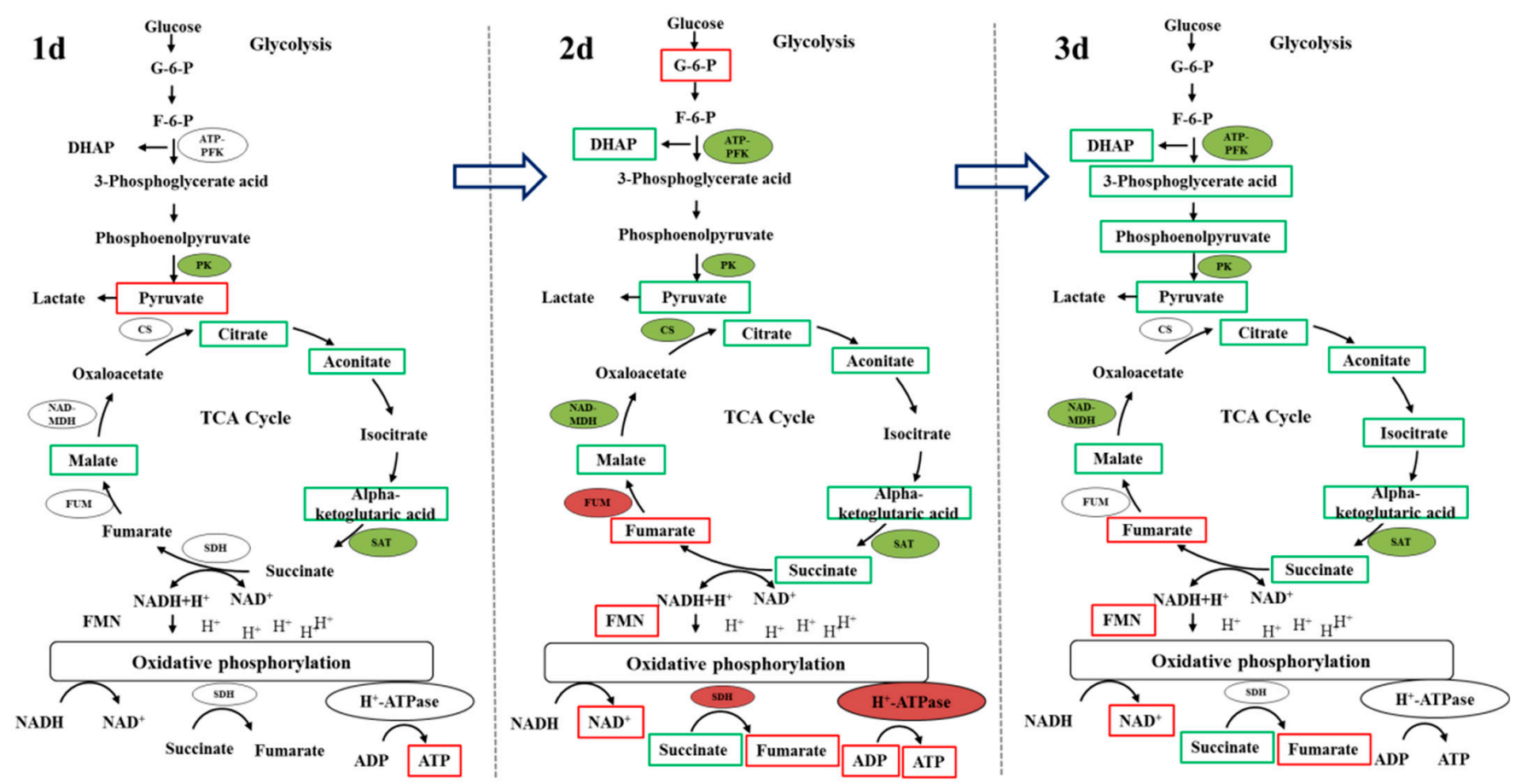

Figure 8. An overview of changes in energy metabolism in carbon starvation-induced fruitlet abscission in litchi. Red shading indicates a significant up-regulation, green shading indicates a significant down-regulation. $1 \mathrm{~d}, 2 \mathrm{~d}$ and $3 \mathrm{~d}$ represent the days after GPD (girdling plus defoliation ) treatment.

Author Contributions: Conceptualization, Q.W., J.L. and M.Z.; methodology, H.W.; Software, X.M. and Y.Y.; resources, X.H.; Operation and investigation, Q.W. and Q.C.; writing-original draft, Q.W.; writing-review and editing, J.L., M.Z. and X.M. All authors have read and agreed to the published version of the manuscript.

Funding: This work was supported by grants from the Laboratory of Lingnan Modern Agriculture Project (NZ NT2021004 ), the National Natural Science Foundation of China (32072514) and the State Key Laboratory for Conservation and Utilization of Subtropical Agro-bioresources (SKLCUSAa201921).

Informed Consent Statement: Informed consent was obtained from all subjects involved in the study.

Data Availability Statement: All the data are already provided in the main manuscript. Contact the corresponding author if further explanation is required.

Conflicts of Interest: The authors declare no conflict of interest.

\section{References}

1. Zhao, M.L.; Li, J.G. Molecular events involved in fruitlet abscission in litchi. Plants 2020, 9, 151. [CrossRef] [PubMed]

2. Megguer, C.A.; Fugate, K.K.; Lafta, A.M.; Ferrareze, J.P.; Deckard, E.L.; Campbell, L.G.; Lulaie, C.; Finger, F.L. Glycolysis is dynamic and relates closely to respiration rate in stored sugarbeet roots. Front. Plant Sci. 2017, 8, 861. [CrossRef]

3. Omena-Garcia, R.P.; Araújo, W.L.; Gibon, Y.; Fernie, A.R.; Nunes-Nesi, A. Measurement of tricarboxylic acid cycle enzyme activities in plants. In Plant Respiration and Internal Oxygen; Humana Press: New York, NY, USA, 2017; Volume 1670.

4. Kadenbach, B. Intrinsic and extrinsic uncoupling of oxidative phosphorylation. Biochim. Biophys. Acta-Bioenerg. 2003, 1604, 77-94. [CrossRef]

5. Fernie, A.R.; Carrari, F.; Sweetlove, L.J. Respiratory metabolism: Glycolysis, the TCA cycle and mitochondrial electron transport. Curr. Opin. Plant Biol. 2004, 7, 254-261. [CrossRef] [PubMed]

6. Millar, A.H.; Whelan, J.; Soole, K.L.; Day, D.A. Organization and regulation of mitochondrial respiration in plants. Annu. Rev. Plant Biol. 2011, 62, 79-104. [CrossRef]

7. O'leary, B.; Plaxton, W.C. Multifaceted functions of post-translational enzyme modifications in the control of plant glycolysis. Curr. Opin. Plant Biol. 2020, 55, 28-37. [CrossRef] [PubMed] 
8. Liu, L.; Wang, B.; Liu, D.; Zou, C.L.; Wu, P.R.; Wang, Z.Y.; Wang, Y.B.; Li, C.F. Transcriptomic and metabolomic analyses reveal mechanisms of adaptation to salinity in which carbon and nitrogen metabolism is altered in sugar beet roots. BMC Plant Biol. 2020, 20, 615-620. [CrossRef] [PubMed]

9. Ye, T.T.; Shi, H.T.; Wang, Y.P.; Yang, F.; Chan, Z.L. Contrasting proteomic and metabolomic responses of bermudagrass to drought and salt stresses. Front. Plant Sci. 2016, 7, 1694. [CrossRef] [PubMed]

10. Huang, C.Y.; Roessner, U.; Eickmeier, I.; Genc, U.; Callahan, D.L.; Shirley, N.; Langridge, P.; Bacic, A. Metabolite profiling reveals distinct changes in carbon and nitrogen metabolism in phosphate-deficient barley plants (Hordeum vulgare L.). Plant Cell Physiol. 2008, 49, 691. [CrossRef]

11. Guo, R.; Zhou, J.; Yang, F.; LI, F.; LI, H.R.; Xia, X.; Liu, Q. Growth metabolism of wheat under drought stress at the jointing-booting stage. Chin. J. Plant Ecol. 2016, 40, 1319-1327.

12. Li, D.; Limwachiranon, J.; Li, L.; Du, R.X.; Luo, Z.S. Involvement of energy metabolism to chilling tolerance induced by hydrogen sulfide in cold-stored banana fruit. Food Chem. 2016, 208, 272-278. [CrossRef] [PubMed]

13. Zhao, H.D.; Jiao, W.X.; Cui, K.B.; Fan, X.G.; Shu, C.; Zhang, W.L.; Cao, J.K.; Jiang, W.B. Near-freezing temperature storage enhances chilling tolerance in nectarine fruit through its regulation of soluble sugars and energy metabolism. Food Chem. 2019, 289, 426-435. [CrossRef] [PubMed]

14. Zhou, C.J.; Lakso, A.N.; Robinson, T.L.; Gan, S.S. Isolation and characterization of genes associated with shade-induced apple abscission. Mol. Genet. Genom. 2008, 280, 83-92. [CrossRef] [PubMed]

15. Domingos, S.; Scafidi, P.; Cardoso, V.; Leitao, A.E.; Di, L.R.; Oliveira, C.M.; Goulao, L.F. Flower abscission in Vitis vinifera L. triggered by gibberellic acid and shade discloses differences in the underlying metabolic pathways. Front. Plant Sci. 2015, 6, 457. [CrossRef] [PubMed]

16. Yang, Z.Q.; Li, M.; Zhang, X.Y.; Yu, Y.; Wang, H.C.; Huang, X.M. Effects of starvation stress on fruit abscission and sugar metabolism in longan. J. Fruit Sci. 2011, 28, 428-432.

17. Kuang, J.; Wu, J.Y.; Zhong, H.Y.; Li, C.Q.; Chen, J.Y.; Lu, W.J.; Li, J.G. Carbohydrate stress affecting fruitlet abscission and expression of genes related to auxin signal transduction pathway in litchi. Int. J. Mol. Sci. 2012, 13, 16084-16103. [CrossRef] [PubMed]

18. Domingos, S.; Fino, J.; Cardoso, V.; Sánchez, C.; Ramalho, J.C.; Larcher, R.; Paulo, O.S.; Oliveira, C.M.; Goulao, L.F. Shared and divergent pathways for flower abscission are triggered by gibberellic acid and carbon starvation in seedless Vitis vinifera L. BMC Plant Biol. 2016, 16, 38. [CrossRef]

19. Li, C.Q.; Wang, Y.; Huang, X.M.; Li, J.; Wang, H.C.; Li, J.G. An improved fruit transcriptome and the identification of the candidate genes involved in fruit abscission induced by carbohydrate stress in litchi. Front. Plant Sci. 2015, 6, 439. [CrossRef]

20. Wang, S.T.; Li, X.N.; Wang, J.; Ling, X.M. Metabonomics and its analytical technique. Chin. J. Pharm. Anal. 2010, 30, $1792-1799$.

21. Jenner, H.L.; Winning, B.M.; Millar, A.H.; Tomlinson, K.L.; Leaver, C.J.; Hill, S.A. NAD Malic enzyme and the control of carbohydrate metabolism in potato tubers. Plant Physiol. 2001, 126, 1139-1149. [CrossRef] [PubMed]

22. Burrell, M.M.; Mooney, P.J.; Blundy, M.; Carter, D.; Wilson, F.; Green, J.; Blundy, K.S.; Rees, T. Genetic manipulation of 6phosphofructokinase in potato tubers. Planta 1994, 194, 95-101. [CrossRef]

23. Danson, M.J.; Black, S.C.; Woodland, D.L.; Wood, P.A. Citric acid cycle enzymes of the archaebacteria: Citrate synthase and succinate thiokinase. FEBS Lett. 1985, 179, 120-124. [CrossRef]

24. Schirawski, J.; Unden, G. Anaerobic respiration of bacillus macerans with fumarate, TMAO, nitrate and nitrite and regulation of the pathways by oxygen and nitrate. Arch. Microbiol. 1995, 163, 148-154. [CrossRef]

25. Zhang, J.H.; Liu, Y.P.; Pan, Q.H.; Zhan, J.C.; Wang, X.Q.; Huang, W.D. Changes in membrane-associated $\mathrm{H}^{+}$-ATPase activities and amounts in young grape plants during the cross adaptation to temperature stresses. Plant Sci. 2006, 170, 768-777. [CrossRef]

26. Zhong, H.Y.; Chen, J.W.; Li, C.Q.; Chen, L.; Wu, J.Y.; Chen, J.Y.; Liu, W.J.; Li, J.G. Selection of reliable reference genes for expression studies by reverse transcription quantitative real-time PCR in litchi under different experimental conditions. Plant Cell Rep. 2011, 30, 641-653. [CrossRef] [PubMed]

27. Yuan, R.C.; Huang, H.B. Litchi fruit abscission: Its patterns, effect of shading and relation to endogenous abscisic acid. Sci. Hortic. 1988, 36, 281-292. [CrossRef]

28. Xiang, X.; Zhang, Z.W.; Qiu, Y.P.; Yuan, P.Y.; Wang, B.Q. The relationship between fruit setting and endogenous hormones in 'Nuomici' litchi. Acta Hortic. Sin. 1994, 1, 1-6.

29. Zhou, X.J.; Huang, D.Y.; Huang, H.B.; Wu, D.Y. Effect of the spiral girdling made on fruit setting, carbohydrate and hormone on 'Nuomici' litchi fruit abscission. Acta Hortic. Sin. 1999, 26, 77-80.

30. Mehouachi, J.; Serna, D.; Zaragoza, S.; Agustib, M.; Talona, M.; Primo-Milloa, E. Defoliation increases fruit abscission and reduces carbohydrate levels in developing fruits and woody tissues of Citrus unshiu. Plant Sci. 1995, 107, 189-197. [CrossRef]

31. Givan, C.V. Evolving concepts in plant glycolysis: Two centuries of progress. Biol. Rev. 1999, 74, 277-309. [CrossRef]

32. Plaxton, W.C.; Podestá, F.E. The functional organization and control of plant respiration. Crit. Rev. Plant Sci. 2006, 25, 159-198. [CrossRef]

33. Sweetlove, L.J.; Beard, K.F.; Nunes-Nesi, A.; Fernie, A.R.; Ratcliffe, R.G. Not just a circle: Flux modes in the plant TCA cycle. Trends Plant Sci. 2010, 15, 462. [CrossRef] [PubMed]

34. Yang, F.Y. The mechanism of oxidative phosphorylat about recent studies. Bull. Biol. 1988, 9, $16-18$. 